[0212-7199 (2007) 24: 11; pp 525-530] ANALES DE MEDICINA INTERNA Copyright (C) 2007 ARAN EDICIONES, S.L.

AN. MED. INTERNA (Madrid) Vol. 24, N. ${ }^{\circ} 11$, pp. 525-530, 2007

\title{
Comorbilidad, pluripatología, consumo de recursos y pronóstico de pacientes ingresados en una unidad de Medicina Interna
}

\author{
F. MEDRANO GONZÁLEZ, M. MELERO BASCONES, M. A. BARBA ROMERO, \\ J. GÓMEZ GARRIDO, J. LLABRÉS DÍAZ, J. MORENO SALCEDO
}

Servicio de Medicina Interna. Hospital Universitario. Albacete

COMORBIDITY, PLURIPATHOLOGY, RESOURCE USE AND PROGNOSIS OF PATIENTS HOSPITALIZED IN INTERNAL MEDICINE AREAS

\section{RESUMEN}

Objetivo: Comparar la definición de paciente con pluripatología (PP) del Servicio de Salud de Andalucía con el índice de comorbilidad de Charlson (IC) respecto al consumo de recursos y pronóstico de enfermos hospitalizados.

Pacientes y métodos: Estudio prospectivo observacional de 207 pacientes ingresados de forma consecutiva en una unidad de medicina interna en los que se determinó la presencia de PP, una variante de PP obtenida de aplicar al menos tres categorías clínicas en lugar de dos (PP3), el IC y su valor ajustado con la edad (ICE), y la relación de estas variables con el consumo de recursos (atenciones urgentes, consultas programadas de especialidades y episodios de hospitalización en los doce meses previos, duración de la estancia, procedimientos propios, procedimientos de otras especialidades e interconsultas) y pronóstico (mortalidad intrahospitalaria, deterioro funcional significativo y reingreso precoz).

Resultados: La frecuencia de PP fue de 40,6\% y la de PP3 de 16,9\%. La mediana de la puntuación del IC fue de 2 (0-7) y la del ICE de 4 (011). Se detectaron las siguientes relaciones independientes: IC de 3 o mayor con reingreso precoz y duración de la estancia; ICE de 5 o mayor con la mortalidad; PP3 con la mortalidad, reingreso precoz y con urgencias, ingresos y consultas en el último año; categorías B y F de PP con las hospitalizaciones en el último año; categoría D de PP con las consultas en el último año; y la asociación de categorías A, B y C con la mortalidad y hospitalizaciones en el último año. PP no se relacionó de forma independiente con ninguna variable.

Conclusión: El IC y la definición de PP no identifican la misma población de pacientes hospitalizados. Modificaciones de la definición de PP, como PP3, o el análisis de las distintas categorías clínicas de PP y sus asociaciones, podrían mejorar la utilidad de este concepto.

PALABRAS CLAVE: Pluripatología. Comorbilidad. Consumo de recursos. Pronóstico.

\section{ABSTRACT}

Objective: To compare the concept of patient with pluripathology $(P P)$ with the index of comorbidity of Charlson (IC) respect to the resources use and prognosis of hospitalized patients.

Patients and methods: An observational prospective study of 207 consecutively hospitalized patients in an internal medicine unit was conducted. The PP, a variant of PP with three or more criteria (PP3), IC and IC fit to the age (ICE) were determined, and their relation with the consumption of resources (emergency attentions, programmed consultations of specialties and episodes of hospitalization in the last year, length of stay, own procedures, other specialty procedures and consulting) and prognosis (hospital mortality, significant functional deterioration and rehospitalization) were stayed.

Results: The frequency of PP and PP3 was 40.6 and 16.9\%, respectively. The median of the IC and ICE score were $2(0-7)$ and $4(0-11)$, respectively. We found the following independent relations: IC of 3 or greater with the rehospitalization and the length of stay; ICE of 5 or greater with mortality; PP3 with mortality, rehospitalization and emergency visits, hospitalizations and consultations in the last year; the categories $B$ and $F$ of PP with hospitalizations in the last year; the category $D$ of $P P$ with consultations in the last year; and the association of categories $A, B$ and $C$ with mortality and hospitalizations in the last year. PP was not related in an independent form with any variable.

Conclusion: The IC and the concept of PP do not identify the same hospitalized patient population. Modifications of the PP definition, like $P P 3$, or the analysis of the different clinical categories of PP and their associations, could improve the utility of this concept.

KEY WORDS: Comorbidity. Health resources. Prognosis.

Medrano González F, Melero Bascones M, Barba Romero MA, Gómez Garrido J, Llabrés Díaz J, Moreno Salcedo J. Comorbilidad, pluripatología, consumo de recursos y pronóstico de pacientes ingresados en una unidad de Medicina Interna. An Med Interna (Madrid) 2007; 24: 525-530.

\section{INTRODUCCIÓN}

La importancia de la comorbilidad se debe a su alta prevalencia, especialmente en pacientes ancianos, y su impacto en la salud y los cuidados sanitarios (1). Entendida como la pre- sencia de una o más enfermedades entre sujetos con una enfermedad índice, se suele medir por la suma del número de enfermedades presentes en un individuo, por medio de escalas de comorbilidad que combinan el número y gravedad de las enfermedades presentes (1), de las cuales la más utilizada y

Trabajo aceptado: 9 de julio de 2007 
validada es el índice de comorbilidad de Charlson (2) (IC), o bien registrando las asociaciones de enfermedades concretas que aparecen en determinados pacientes. La reciente definición de paciente con pluripatología (PP) del Servicio de Salud de Andalucía (3) podría incluirse dentro de esta última forma de medir la comorbilidad.

Varios estudios de PP hospitalizados $(4,5)$ realizados en nuestro país, han puesto de manifiesto la importancia de dicho concepto como indicador de un grupo de pacientes de especial fragilidad clínica que provoca un mayor consumo de recursos y disminución de la situación funcional.

Existen pocos estudios que exploren la frecuencia de PP en áreas de medicina interna y, en nuestro conocimiento, no se ha comparado con otras formas de medir la comorbilidad, por lo que realizamos un estudio prospectivo aplicando y comparando el concepto de PP y el IC en pacientes ingresados en una unidad de medicina interna.

\section{PACIENTES Y MÉTODO}

Estudio prospectivo observacional de todos los pacientes ingresados desde el 1 de octubre al 31 de diciembre de 2005 en una unidad de medicina interna de 30 camas del Hospital Universitario de Albacete, complejo hospitalario que asiste a una población de 360.000 habitantes.

Se recogió el diagnóstico principal según CIE-9-MC, la situación funcional basal, al ingreso y al alta según la escala de Barthel (6), la presencia de PP aplicando los criterios de la Consejería de Salud de Andalucía (3) y una modificación de la misma obtenida de aplicar al menos tres categorías clínicas en lugar de dos (PP3), el IC clásico y el IC ajustado a la edad (2). El consumo de recursos se valoró con medidas del proceso de cuidados: número de atenciones urgentes, consultas programadas de especialidades y episodios de hospitalización en los doce meses previos, duración de la estancia, número de procedimientos propios realizados por nuestra especialidad o por otras especialidades y número de interconsultas durante la hospitalización. El pronóstico se midió con variables de resultado: mortalidad intrahospitalaria, deterioro funcional significativo y reingreso precoz. Se consideró que existía alteración de la situación funcional cuando el paciente presentaba una escala de Barthel menor de 60, deterioro funcional significativo si apareció un descenso de 10 o más puntos entre los valores basales y al alta de la misma escala y reingreso precoz cuando ocurrió en los 31 días siguientes al alta por el mismo motivo o relacionado.

Se realizó un análisis multivariante con un modelo explicativo y todas las variables dicotómicas. Las variables independientes fueron: PP o PP3, las categorías clínicas de PP y las asociaciones más frecuentes de las mismas, IC agrupado en valores de 3 o más (IC3) o menos de 3, ICE agrupado en valores de 5 o más (ICE5) o menos de 5 y el diagnóstico principal según los grupos de la clasificación CIE-9-MC. Las variables dependientes fueron: ingresos, consultas y urgencias en el último año, duración de la estancia, procedimientos realizados por nuestra especialidad, procedimientos realizados por otras especialidades e interconsultas, todos ellos con valores dicotómicos por encima o igual o inferior a la mediana; mortalidad intrahospitalaria, deterioro funcional significativo y reingreso precoz.

\section{ANÁLISIS ESTADÍSTICO}

Las variables cuantitativas se expresan, dependiendo de su distribución, como media (desviación estándar) o mediana (intervalo) y las cualitativas como porcentaje. La comparación entre variables continuas se realizó con la prueba Anova o la de Kruskal-Wallis y entre variables categóricas con la prueba de ji cuadrado mediante la corrección de Yates y, cuando fue preciso, la prueba exacta de Fisher. El análisis multivariante se realizó por regresión logística múltiple con el método enter. Para los cálculos se han utilizado los programas Epi Info 6.04a y SPSS versión 13.

\section{RESULTADOS}

Se incluyeron 207 pacientes de 72 (22-92) años de edad, $48 \%$ mujeres, $1 \%$ institucionalizados y procedentes de Urgencias en el $85 \%$. La duración de la estancia fue de 10 (2-54) días. El número de ingresos, atenciones urgentes y consultas de especialidades en el último año fueron de 0 (0-7), 2 (0-12) y 4 (0-28), respectivamente. La escala de Barthel basal fue de 95 (0-100), al ingreso de 85 (0-100) y al alta de 92,5 (0-100). Presentaban alteración funcional basal, al ingreso y al alta el 15,29 y $17 \%$, respectivamente, y apareció deterioro funcional significativo en el $18 \%$. El número de procedimientos de nuestra especialidad realizados durante la hospitalización fue de 0 (0-2), los solicitados a otras especialidades de 1 (0-7) y de interconsultas de 0 (0-2). La mortalidad hospitalaria fue de $11.1 \%$ y hubo reingreso precoz en el $12 \%$.

Los diagnósticos principales estaban incluidos en los grupos de la clasificación CIE-9-MC siguientes: aparato circulatorio (27\%), aparato respiratorio (17\%), aparato digestivo (11\%), neoplasias $(8 \%)$, sistema endocrino y metabólico (7\%), síntomas y signos mal definidos $(6 \%)$, aparato genitourinario $(5 \%)$, infecciones o parasitosis (5\%), sistema osteomuscular $(3 \%)$, piel $(3 \%)$, sangre $(2 \%)$, sistema nervioso (2\%), traumatismos y envenenamientos $(2 \%)$ y trastornos mentales $(1 \%)$.

El IC fue de 2 (0-7), el ICE de 4 (0-11) y presentaban IC3 el 32,9 e ICE5 el 49,8\%. La frecuencia de las variables medidas en los pacientes con IC3 e ICE5 se señala en la tabla I. En resumen, ambos grupos de pacientes presentaban mayor edad, peor situación funcional basal, mayor número de ingresos y urgencias en el último año, menos procedimientos de otras especialidades y peor situación funcional al alta, aunque no mayor deterioro funcional significativo. Además, el grupo IC3 se relacionaba con un diagnóstico principal perteneciente al grupo de enfermedades del aparato digestivo, mayor duración de la estancia y más reingresos, y el grupo ICE5 con diagnóstico principal perteneciente a los grupos de enfermedades del aparato circulatorio o respiratorio y mayor mortalidad.

La frecuencia de PP fue de 40,6\% y la de PP3 de 16,9\%. La frecuencia de las variables medidas en los pacientes con PP y PP3 se señala en la tabla II. En resumen, ambos grupos de pacientes presentaban mayor edad, peor situación funcional basal y al ingreso, mayor frecuencia de un diagnóstico principal perteneciente al grupo de enfermedades del aparato circulatorio, mayor número de ingresos y de consultas en el último año y menos procedimientos de otras especialidades. Además, en el grupo de pacientes PP3 fueron mayores el número de urgencias, la mortalidad y el deterioro funcional significativo, con peor situación funcional al alta. 
TABLA I

COMPARACIÓN DE LOS PACIENTES SEGÚN LOS VALORES DEL ÍNDICE DE CHARLSON

\begin{tabular}{|c|c|c|c|c|c|c|}
\hline & \multicolumn{3}{|c|}{$\begin{array}{l}\text { Pacientes con indice de Charlson } \\
\text { igual o mayor de } 3 \text { (IC3) }\end{array}$} & \multicolumn{3}{|c|}{$\begin{array}{l}\text { Pacientes con índice de Charlson ajustado } \\
\text { a la edad igual o mayor de } 5 \text { (ICE5) }\end{array}$} \\
\hline & $\begin{array}{c}\text { Sí } \\
(n=68)\end{array}$ & $\begin{array}{c}\text { No } \\
(n=139)\end{array}$ & $\begin{array}{c}P \\
\text { OR (IC95\%) }\end{array}$ & $\begin{array}{c}\text { Si } \\
(n=103)\end{array}$ & $\begin{array}{c}\text { No } \\
(n=104)\end{array}$ & $\begin{array}{c}P \\
\text { OR (IC95\%) }\end{array}$ \\
\hline $\begin{array}{l}\text { Edada }^{a} \\
\text { Diagnóstico principal (\%) }\end{array}$ & $75,5(25-91)$ & $70(22-92)$ & 0,001 & $78(25-92)$ & $65(22-80)$ & 0,000 \\
\hline Enfermedad infecciosa o parasitaria & 0 & 7 & $0,6(0,5-0,7)^{*}$ & 2 & 8 & $0,2(0-1,1)$ \\
\hline Enfermedad de la sangre & 0 & 4 & $0,6(0,6-0,7)^{*}$ & 0 & 5 & $0,4(0,4-0,5)$ \\
\hline Enfermedad del aparato circulatorio & 34 & 24 & $1,6(0,8-3,1)$ & 35 & 19 & $2,2(1,1-4,2)^{*}$ \\
\hline Enfermedad del aparato respiratorio & 19 & 16 & $1,2(0,5-2,6)$ & 23 & 11 & $2,5(1,1-5,5)^{*}$ \\
\hline \multicolumn{7}{|l|}{ Pluripatología (\%) } \\
\hline Ppb & 79 & 22 & $14(6,8-28,6)^{*}$ & 67 & 14 & $12(6-23,8)^{b}$ \\
\hline PP3 & 40 & 6 & $10,7(4,5-25)^{*}$ & 32 & 2 & $24(5,5-103)^{b}$ \\
\hline Categorías $A, B$ y $C^{d}$ & 7 & 4 & $1,7(0,5-5,9)$ & 9 & 2 & $4,8(1,02-23,1)^{b}$ \\
\hline \multicolumn{7}{|l|}{ Situación funcional } \\
\hline Escala de Barthel basal ${ }^{a}$ & $85(0-100)$ & $95(0-100)$ & 0,01 & $90(0,100)$ & $100(0-100)$ & 0,009 \\
\hline Alteración funcional basal (\%) ${ }^{e}$ & 22 & 11 & $2,3(0,8-6)$ & 20 & 8 & $2,8(0,9-8,2)$ \\
\hline Escala de Barthel al ingresoa & $65(0-100)$ & $85(0-100)$ & 0,000 & $65(0-100)$ & $90(0-100)$ & 0,000 \\
\hline Alteración funcional al ingreso $(\%)^{e}$ & 44 & 20,5 & $3,1(1,3-6,9)$ & 41 & 17 & $3,3(1,4-7,7)^{*}$ \\
\hline Escala de Barthel al alta & $85(0-100)$ & $95(0-100)$ & 0,03 & $85(0-100)$ & $100(0-100)$ & 0,01 \\
\hline Alteración funcional al alta $(\%)^{e}$ & 23 & 14 & $1,8(0,6-4,9)$ & 22 & 12,5 & $2(0,7-5,5)$ \\
\hline Deterioro funcional significativo (\%) & 26 & 14 & $2,1(0,7-5,6)$ & 24 & 12,5 & $2,2(0,8-6)$ \\
\hline Mortalidad intrahospitalaria (\%) & 13,2 & 10,1 & $1,2(0,5-3,3)$ & 18,4 & 3,8 & $5,6(1,8-17)^{*}$ \\
\hline Reingreso precoz $(\%)$ & 21 & 7 & $3,3(1,3-7,9)$ & 15,5 & 8 & $2.2(0,9-5,4)$ \\
\hline \multicolumn{7}{|l|}{ Consumo de recursos durante el último año (\%) } \\
\hline Una o más hospitalizaciones & 51,5 & 30 & $2,4(1,3-4,4)$ & 44 & 31 & $1,7(0,9-3)$ \\
\hline Cinco o más consultas de especialidades & 53 & 42 & $1,5(0,8-2,7)$ & 48,5 & 43 & $1,2(0,7-2,1)$ \\
\hline Tres o más atenciones en Urgencias & 47 & 35 & $1,6(0,9-2,9)$ & 43 & 36 & $1,3(0,7-2,3)$ \\
\hline \multicolumn{7}{|l|}{ Consumo de recursos durante la hospitalización } \\
\hline Estancia hospitalaria & $11,5(2-44)$ & $10(2-54)$ & 0,08 & $11(2-54)$ & $10(2-44)$ & 0,3 \\
\hline Uno o más procedimientos propios (\%) & 25 & 27 & $0,8(0,4-1,7)$ & 23 & 30 & $0,7(0,3-1,3)$ \\
\hline Dos o más procedimientos de otras especialidades (\%) & 31 & 44 & $0,5(0,3-1)$ & 32 & 47 & $0,5(0,3-1,3)^{*}$ \\
\hline Una o más interconsultas (\%) & 32 & 28 & $1,2(0,6-2,2)$ & 24 & 35 & $0,6(0,3-1,1)$ \\
\hline
\end{tabular}

a) Variable expresada como mediana (intervalo); b) Pacientes con dos o más categorías clínicas de pluripatología; c) Pacientes con tres o más categorías clínicas de pluripatología; d) Asociación de categorías A, B y C de pluripatología; e) Escala de Barthel menor de 60 puntos; * p < 0,05.

Las distintas categorías clínicas, sus asociaciones más frecuentes y la frecuencia de las variables del consumo de recursos y pronóstico de cada una de ellas se señalan en la tabla III.

En el análisis multivariante se detectaron las siguientes relaciones independientes: diagnóstico principal de neoplasia con la mortalidad (OR 7,1: 1,9-26,6), diagnóstico principal de enfermedad endocrinometabólica con una duración de la estancia mayor de 10 días (OR 0,1: 0,01-0,8), diagnóstico principal de enfermedad del aparato circulatorio con uno o más ingresos en el último año (OR 2,6: 1,1-6,0) y diagnóstico principal de enfermedad del aparato digestivo (OR 4,1: 1,610,4) o músculoesquelético (OR 7.1: 1.3-38.8) con uno o más procedimientos propios de nuestra especialidad; las categorías clínicas B (OR 7,2: 1,2-43,1) y F (OR 3,7: 1,09-12,9) de PP con uno o más ingresos en el último año; la categoría D de PP con cinco o más consultas de especialidades en el último año (OR 4,1: 1,05-16,1); la asociación ABC con la mortalidad (OR 5,8: 1,3-25) y con uno o más ingresos en el último año (OR 22,3: 1,06-466,7); un IC3 con el reingreso precoz (OR
2,8: 1,1-7,2) y una estancia de más de 10 días (OR 2: 1,1-3,7); un ICE5 con la mortalidad (OR 4: 1,1-13,5); y PP3 con la mortalidad (OR 3,6: 1,2-10,5), con uno o más ingresos en el último año (OR 3,6: 1,5-8,6), tres o más urgencias en el último año (OR 4.4: 2.0-9.6) y con cinco o más consultas de especialidades en el último año (OR 3,5: 1,6-7,9). El PP no se relaciona de forma independiente con ninguna de las variables.

\section{DISCUSIÓN}

La definición de PP va implícitamente unida al concepto de comorbilidad, pero una diferencia importante entre el PP y el IC es que en el PP se incluye el diagnóstico principal, otros diagnósticos secundarios y la situación funcional del paciente, mientras que en el IC sólo se tienen en cuenta los diagnósticos secundarios y no el diagnóstico principal, salvo que el motivo de ingreso sea un proceso crónico agudizado, 
TABLA II

COMPARACIÓN DE LOS PACIENTES SEGÚN LA PLURIPATOLOGÍA

\begin{tabular}{|c|c|c|c|c|c|c|}
\hline & \multicolumn{3}{|c|}{$\begin{array}{l}\text { Pacientes con dos o más categorías } \\
\text { de pluripatología (PP) }\end{array}$} & \multicolumn{3}{|c|}{$\begin{array}{l}\text { Pacientes con tres o más categorías } \\
\text { de pluripatología (PP3) }\end{array}$} \\
\hline & $\begin{array}{c}\text { Si } \\
(n=84)\end{array}$ & $\begin{array}{c}\text { No } \\
(n=123)\end{array}$ & $\begin{array}{c}P \\
\text { OR (IC95\%) }\end{array}$ & $\begin{array}{c}\text { Si } \\
(n=35)\end{array}$ & $\begin{array}{c}\text { No } \\
(n=172)\end{array}$ & $\begin{array}{c}P \\
\text { OR (IC95\%) }\end{array}$ \\
\hline $\begin{array}{l}\text { Edad } \\
\text { Diagnóstico principal (\%) }\end{array}$ & $75(33-91)$ & $68(22-92)$ & 0,000 & $76(57-89)$ & $70(22-92)$ & 0,001 \\
\hline $\begin{array}{l}\text { Enfermedad del aparato circulatorio } \\
\text { Comorbilidad }\end{array}$ & 43 & 16 & $3,8(2-7,3)^{*}$ & 51 & 22 & $3,7(1,7-7,9)^{*}$ \\
\hline Indice de Charlson ${ }^{a}$ & $3(0-8)$ & $1(0-6)$ & 0,000 & $4(1-8)$ & $1(0-7)$ & 0,000 \\
\hline Indice de Charlson igual o mayor de 3 (\%) & 64 & 11 & $14(6,8-28,6)^{*}$ & 77 & 24 & $10,7(4,5-25)^{*}$ \\
\hline $\begin{array}{l}\text { Indice de Charlson ajustado edad } \\
\text { Indice de Charlson ajustado a la edad igual o mayor de } 5(\%)\end{array}$ & $\begin{array}{c}6(0-11) \\
82\end{array}$ & $\begin{array}{c}3(0-9) \\
28\end{array}$ & $\begin{array}{c}0,000 \\
12(6-23,8)^{*}\end{array}$ & $\begin{array}{l}7(4-11) \\
94\end{array}$ & $\begin{array}{l}4(0-10) \\
41\end{array}$ & $\begin{array}{c}0,000 \\
24(5,5-103)^{*}\end{array}$ \\
\hline \multicolumn{7}{|l|}{ Situación funcional } \\
\hline Escala de Barthel basal ${ }^{2}$ & $90(0-100)$ & $100(0-100)$ & 0,02 & $80(20-100)$ & $95(0-100)$ & 0,001 \\
\hline Alteración funcional basalb (\%) & 19 & 11 & $1,9(0,7-5,2)$ & 32 & 11 & $3,8(1,3-10,8)^{*}$ \\
\hline Escala de Barthel al ingreso ${ }^{a}$ & $70(0-100)$ & $85(0-100)$ & 0,02 & $50(0-100)$ & $85(0-100)$ & 0,000 \\
\hline Alteración funcional al ingreso ${ }^{b}(\%)$ & 35 & 25 & $1,6(0,7-3,6)$ & 57 & 23,5 & $4,3(1,6-11,5)^{*}$ \\
\hline Escala de Barthel al alta ${ }^{a}$ & $90(0-100)$ & $95(0-100)$ & 0,1 & $60(0-100)$ & $95(0-100)$ & 0,002 \\
\hline Alteración funcional al altab $(\%)$ & 21 & 14,5 & $1,5(0,5-4,1)$ & 33 & 15 & $2,8(0,8-9,7)$ \\
\hline Deterioro funcional significativo (\%) & 21 & 16 & $1,3(0,5-3,6)$ & 40 & 15 & $3,8(1,1-12,5)^{*}$ \\
\hline Mortalidad intrahospitalaria (\%) & 14,3 & 8,9 & $1,6(0,7-4)$ & 28,6 & 7,6 & $4,8(1,9-12,3)^{*}$ \\
\hline Reingreso precoz $(\%)$ & 14 & 10 & $1,5(0,6-3,6)$ & 20 & 10 & $2,2(0,8-6)$ \\
\hline \multicolumn{7}{|l|}{ Consumo de recursos durante el último año (\%) } \\
\hline Una o más hospitalizaciones & 49 & 29 & $2,3(1,2-4,1)^{*}$ & 69 & 31 & $4,8(2,2-10,7)^{*}$ \\
\hline Cinco o más consultas de especialidades & 52 & 41,5 & $1,5(0,8-2,7)$ & 71 & 41 & $3,6(1,6-8)^{*}$ \\
\hline $\begin{array}{l}\text { Consumo de recursos durante la hospitalización } \\
\text { Estancia hospitalaria }{ }^{2}\end{array}$ & $10,5(2-54)$ & $10(2-43)$ & 0,5 & $10(2-29)$ & $10(2-54)$ & 0,8 \\
\hline Uno o más procedimientos propios $(\%)$ & 23 & 29 & $0,7(0,3-1,3)$ & 29 & 26 & $1,1(0,5-2,5)$ \\
\hline Dos o más procedimientos de otras especialidades (\%) & 29 & 47 & $0,4(0,2-0,8)^{*}$ & 29 & 42 & $0,5(0,2-1,2)$ \\
\hline Una o más interconsultas ( $\%$ ) & 23 & 34 & $0,5(0,2-1)$ & 26 & 30 & $0,7(0,3-1,8)$ \\
\hline
\end{tabular}

a) Variable expresada como mediana (intervalo); b) Escala de Barthel menor de 60 puntos; ${ }^{*} p<0,05$.

y no se considera la situación funcional del paciente. A pesar de ello, la hipótesis principal de este estudio era que la existencia de comorbilidad, medida con el IC, aumentaría el consumo de recursos y empeoraría el pronóstico y que la definición de PP se comportaría de forma similar.

El IC fue diseñado a partir de una cohorte de pacientes ingresados en un hospital de agudos durante un año, incluye distintas categorías a las que se les asigna una determinada puntuación cuya suma expresa la carga de comorbilidad (2). Se ha considerado un método restrictivo que infraestima la prevalencia de comorbilidad, al menos en pacientes mayores con cáncer (7), pero es simple y fácil de usar y es el método más ampliamente utilizado y validado (2) para medir la comorbilidad, hasta el punto que se han realizado adaptaciones para usarlo en bases administrativas de datos con códigos CIE-9-MC $(8,9)$ y existen programas informáticos (10) y páginas de Internet para posibilitar su cálculo. Además, como muchas otras medidas para medir la comorbilidad que se han desarrollado, el IC no solamente sirve para predecir la mortalidad, sino que también se relaciona con la situación funcional y discapacidad de pacientes ancianos (11) e incluso con el consumo de recursos sanitarios $(12,13)$, como ocurre en nuestra muestra de pacientes donde hemos encontrado relación del
IC con la mortalidad, con otra variable de resultado, el reingreso precoz, y con una medida del consumo de recursos como es la duración de la estancia.

Los PP se consideran un grupo de pacientes con unas peculiares necesidades de atención sanitaria que han provocado la elaboración de documentos de consenso sobre su concepto y atención (3). Se ha criticado esta definición por la dificultad de interpretar los criterios de las distintas categorías clínicas, la ausencia de algunos procesos frecuentes en los mismos y la mala concordancia en su aplicación (14). En nuestra muestra de pacientes, la frecuencia de pluripatología, del $41 \%$, fue similar a la descrita por otros autores $(4,5)$, lo que constituye un porcentaje muy importante de los pacientes hospitalizados en medicina interna. Con esta definición hemos identificado a un grupo de pacientes de mayor edad, con mayor deterioro funcional basal y al ingreso, y mayor comorbilidad, pero que no se relaciona de forma independiente con ninguna de las variables que hemos utilizado para medir el consumo de recursos y el pronóstico. Estos datos contrastan con los encontrados por otros autores, que encuentran mayor duración de la estancia y mayor frecuencia de deterioro funcional significativo (4) o mayor mortalidad y mayor número de ingresos y consultas en el último año (5). 
TABLA III

COMPARACIÓN DE LAS CATEGORÍAS CLÍNICAS DE PACIENTE PLURIPATOLÓGICO (PP) Y SUS ASOCIACIONES MÁS FRECUENTES

\begin{tabular}{|c|c|c|c|c|c|c|c|c|c|c|c|}
\hline & \multicolumn{7}{|c|}{ Categorías clínicas de PP } & \multicolumn{4}{|c|}{ Asociaciones de categorías de PP } \\
\hline & $A^{a}$ & $B^{b}$ & $C^{c}$ & $D^{d}$ & $E^{e}$ & $F^{f}$ & $\mathrm{G}^{g}$ & $A C$ & $A B$ & $A F$ & $A B C$ \\
\hline Número de pacientes (\%) & 27,5 & 18 & 24 & 6 & 5 & 10 & 10 & 15,5 & 12 & 7 & 5 \\
\hline \multicolumn{12}{|l|}{ Pronóstico (\%) } \\
\hline Mortalidad intrahospitalaria & 16 & 19 & 14 & 8 & $36^{*}$ & 19 & 15 & 19 & 20 & 27 & $36^{*}$ \\
\hline Reingreso precoz & 16 & 13,5 & 12 & $31^{*}$ & 18 & 14 & 20 & 12,5 & 16 & 13 & 9 \\
\hline Deterioro funcional significativo & 17 & 17 & 25 & 11 & $75^{*}$ & 31 & 33 & 19 & 20 & 12,5 & 50 \\
\hline \multicolumn{12}{|l|}{ Consumo de recursos durante el último año (\%) } \\
\hline Estancia mayor de 10 días & 49 & 40,5 & 35 & 53 & 18 & 43 & 50 & 41 & 36 & 40 & 18 \\
\hline Una o más hospitalizaciones en el último añoh & $49^{*}$ & $67^{*}$ & 47 & 61,5 & 36 & $62^{*}$ & 45 & $56^{*}$ & $68^{*}$ & 60 & $91^{*}$ \\
\hline Cinco o más consultas de especialidades en el último añoh & 53 & $65^{*}$ & 53 & $77^{*}$ & 36 & 52 & 60 & 60 & $68^{*}$ & 47 & $82^{*}$ \\
\hline Tres o más atenciones en Urgencias en el último año ${ }^{h}$ & 46 & $57^{*}$ & 43 & 54 & 54,5 & 57 & 35 & 47 & $68^{*}$ & 47 & $82^{*}$ \\
\hline \multicolumn{12}{|l|}{ Consumo de recursos durante la hospitalización (\%) } \\
\hline Uno o más procedimientos propios' & $16^{*}$ & 27 & 23 & 46 & 27 & 24 & 35 & 19 & 12 & 27 & 18 \\
\hline Dos o más procedimientos de otras especialidades & 30 & 27 & $24,5^{*}$ & 23 & 18 & 38 & 40 & 28 & 28 & 33 & 27 \\
\hline Una o más interconsultas & 21 & 30 & 16 & 23 & 27 & 29 & 25 & $12,5^{*}$ & 28 & 20 & 9 \\
\hline
\end{tabular}

a) Categoría A: insuficiencia cardíca o cardiopatía isquémica; b) Categoría B: enfermedad crónica osteoarticular con limitación funcional o requerimiento de tratamiento continuo, vasculitis, colagenosis o insuficiencia renal crónica; c) Categoría C: limitación crónica al flujo aéreo, asma bronquial, hipoventilación alveolar con limitación funcional o cor pulmonale crónico; d) Categoría D: enfermedad inflamatoria intestinal, hepatopatía crónica activa o sintomática; e) Categoría E: enfermedad neurológica con déficit motor o cognitivo con discapacidad; f) Categoría F: arteriopatía periférica sintomática o diabetes mellitus con repercusión visceral diferente de cardiopatía isquémica; g) Categoría G: enfermedad hematológica u oncológica activa no subsidiaria de tratamiento específico; h) Ingresos hospitalarios, consultas de especialidades y atenciones urgentes en el último año; i) Procedimientos propios de nuestra especialidad; * $\mathrm{p}<0,05$ en la comparación entre la categoría o asociación de categorías y la ausencia de la misma en pacientes con pluripatología.

Las causas de estas discrepancias en los resultados podrían estar relacionadas con las diferencias en la mortalidad y duración de la estancia, y también, como ya se ha señalado en estudios previos (4), con la distinta frecuencia de categorías de PP en los diferentes estudios. Así, la duración de la estancia de nuestros pacientes es mayor y la mortalidad menor que en el grupo de García-Morillo (5), la frecuencia de las categorías $\mathrm{E}$ y $\mathrm{F}$ de nuestros pacientes es menor y la de $\mathrm{G}$ mayor que en la muestra de Zambrana y cols. (4) y la frecuencia de las categorías A, E y F menor y de $\mathrm{C}$ y $\mathrm{G}$ mayor que en el estudio de García-Morillo (5). También son diferentes en los distintos estudios el número de categorías de los pacientes pluripatológicos: en el nuestro, tres o más criterios los cumplían el $16,9 \%$, y en el estudio de García-Morillo (5) en el 35,5\%. Las distintas asociaciones pueden tener no sólo un efecto aditivo, sino sinérgico, al menos en el estado funcional y mental (15), y así, la asociación de las categorías A, B y C, que aparece en el $5 \%$ de nuestros pacientes, confiere una especial gravedad, relacionándose con la mortalidad y con el número de ingresos en el último año.

Si utilizamos el concepto PP3, que aparece en un porcentaje apreciable de pacientes (17\%), la pluripatología sí se relaciona con la mortalidad, reingresos y urgencias, ingresos y consultas en el último año, por lo que parece una medida de utilidad que se puede explorar.

Se ha señalado el papel especial que puede jugar el internista en la atención a estos pacientes con pluripatología (16). En este sentido, hemos encontrado menor utilización de procedimientos de otras especialidades de los pacientes con PP,
IC3 e ICE5, y la de interconsultas de los pacientes con PP, lo que plantea la paradoja de que una mayor complejidad de la asistencia en el paciente ya hospitalizado provoca un menor consumo de recursos que en pacientes no PP, probablemente porque se trata de un subgrupo de pacientes donde los internistas son especialmente competentes, dato que podrían tener en cuenta los gestores sanitarios.

Las principales limitaciones de nuestro estudio se deben al tamaño de la muestra, que aunque es suficiente para el análisis de la mortalidad, puede no tener poder suficiente para encontrar diferencias o asociaciones para otras variables del consumo de recursos o del pronóstico. También hemos perdido poder en el análisis multivariante por la conversión de algunas variables cuantitativas, como IC e ICE, en dicotómicas (IC3 e ICE5), aunque para los objetivos de nuestro estudio y el hecho de tratarse de un modelo explicativo y no predictivo, creemos que ha sido suficiente. Además, no hemos valorado el estado mental, que al igual que el estado funcional debería acompañar a la medida de la comorbilidad, por ser independientes de la misma según los hallazgos de algunos autores (11). También un análisis de costes hubiera enriquecido los resultados de este estudio.

Teniendo en cuenta nuestros resultados creemos necesario mejorar la definición de PP, modificando las características o el número de los criterios empleados en su definición, además de seguir investigando en las distintas categorías individuales y en las asociaciones más frecuentes de las mismas.

En conclusión, el IC y el concepto de PP no identifican la misma población de pacientes respecto al consumo de 
recursos y pronóstico. El IC se relaciona con algunas variables de pronóstico (mortalidad y reingresos) y de consumo de recursos (duración de la estancia) y el concepto de PP no se relaciona con ninguna de las variables estudiadas. Modi- ficaciones de la definición de PP, como PP3, y análisis más detallados de las distintas asociaciones de categorías clínicas de dicha definición, podrían mejorar la utilidad de este concepto.

\section{Bibliografía}

1. Gijsen R, Hoeymans N, Schellevis FG, Ruwaard D, Satariano WA, van den Bos GAM. Causes and consequences of comorbidity: A review. J Clin Epidemiol 2001; 54: 661-74.

2. Charlson ME, Pompei P, Ales KL, McKenzie CR. A new method of classifying prognostic comorbidity in longitudinal studies: Development and validation. J Chron Dis 1987; 40: 373-83.

3. Ollero M, Cabrera JM, de Osorno M, de Villar E, García D, Gómez E, et al. Atención al paciente pluripatológico: Proceso Asistencial Integrado. Sevilla: Consejería de Salud; 2002.

4. Zambrana García JL, Velasco Malagón MJ, Díez García F, Cruz Caparós G, Martín Escalante MD, Adarraga Cansino MD. Características clínicas diferenciales de los enfermos pluripatológicos hospitalizados en servicios de Medicina Interna. Rev Clin Esp 2005; 205: 413-7.

5. García-Morillo JS, Bernabeu-Wittel M, Ollero-Baturone M, Aguilar-Guisad M, Ramírez-Duque N, González de la Puente MA, et al. Incidencia y características clínicas de los pacientes con pluripatología ingresados en una unidad de medicina interna. Med Clin (Barc) 2005; 125: 5-9.

6. Mahoney FI, Barthel DW. Functional Evaluation: The Barthel index. M State Med J 1965; 14: 61-5.

7. Extreman M, Overcash J, Lyman GH, Balducci L. Comorbidity and functional status are independent in older cancer patients. J Clin Oncol 1998; 16: 1582-7.

8. Deyo RA, Cherkin DC, Ciol MA. Adapting a clinical comorbidity index for use with ICD-9-CM administrative databases. J Clin Epidemiol
1992; 45: 613-9.

9. Romano PS, Roos LL, Jollis JG. Adapting a clinical comorbidity index for use with ICD-9-CM administrative data: differing perspectives. J Clin Epidemiol 1993; 46: 1075-9.

10. Hall WH, Ramachandran R, Narayan S, Jani AB, Vijayakumar S. An electronic application for rapidly calculating Charlson comorbidity score. BMC Cancer 2004; 4: 94.

11. Di Bari M, Virgillo A, Matteuzzi D, Inzitari M, Mazzaglia G, Pozzi C, et al. Predictive validity of measures of comorbidity in older community dwellers: The Insufficienza Cardiaca negli Anziani Residenti a Dicomano Study. J Am Geriatr Soc 2006; 54: 210-6.

12. Dominick KL, Dudley TK, Coffman CJ, Bosworth HB. Comparison ot three comorbidity measures for predicting health service use in patients with osteoarthritis. Arthrit Rheum 2005; 53: 666-72.

13. Perkins AJ, Kroenke K, Unutzer J, et al. Common comorbidity scales were similar in their ability to predict health care costs and mortality. J Clin Epidemiol 2004; 57: 1040-8.

14. Pérez-Díaz JM, Martín-Pérez M, Ramos-Cantos MC, San Román-Terán CM. Pacientes con pluripatología ingresados en una unidad de medicina interna. Med Clin (Barc) 2006; 126: 37.

15. Rijken M, Van Kerkhof M, Dekker J, Schellevis G. Comorbidity of chronic diseases. Qual Life Res 2005; 14: 45-55.

16. Sánchez Rodríguez A. El enfermo con pluripatología: La necesidad de una atención integral coordinada. Med Clin (Barc) 2005; 125: 12-3. 